\title{
Standard set of patient-reported outcomes for personality disorder
}

\author{
Valentina Prevolnik Rupel ${ }^{1}$ (1) $\cdot$ Beth Jagger $^{2} \cdot$ Luz Sousa Fialho $^{2} \cdot$ Lisa-Marie Chadderton $^{2} \cdot$ Timea Gintner $^{2}$. \\ Anroud Arntz ${ }^{3}$. Åse-Line Baltzersen ${ }^{4}$. Julia Blazdell ${ }^{5}$. Jan van Busschbach ${ }^{6}$. Marika Cencelli ${ }^{7}$. Andrew Chanen ${ }^{8,20}$. \\ Charlotte Delvaux ${ }^{9} \cdot$ Fieke van Gorp $^{10} \cdot$ Lucie Langford $^{11} \cdot$ Brian McKenna $^{12,19} \cdot$ Paul Moran $^{13} \cdot$ Karla Pacheco $^{14}$. \\ Carla Sharp ${ }^{15} \cdot$ Wei Wang $^{16} \cdot$ Karen Wright $^{17} \cdot$ Mike J. Crawford $^{18}$
}

Accepted: 3 May 2021 / Published online: 2 June 2021

(C) The Author(s) 2021

\begin{abstract}
Purpose The purpose of the article is to present standard set of outcomes for people with personality disorder (PD), in order to facilitate patient outcome measurement worldwide.

Methods The International Consortium for Health Outcomes Measurement (ICHOM) gathered a multidisciplinary international working group, consisting of 16 experts, including clinicians, nurses, psychologists, methodologists and patient representatives, to develop a standard set of outcome measures for people with PD. The Delphi method was used to reach consensus on the scope of the set, outcome domains, outcome measures, case-mix variables and time points for measuring outcomes in service users. For each phase, a project team prepared materials based on systematic literature reviews and consultations with experts.

Results The working group decided to include PD, as defined by International Classification of Diseases 11th revision (ICD11). Eleven core outcomes and three optional outcomes across four health domains (mental health, behaviour, functioning and recovery) were defined as those relevant for people with PD. Validated measures for the selected outcomes were selected, some covering more than one outcome. Case-mix variables were aligned to other ICHOM mental health standard sets and consisted of demographic factors and those related to the treatment that people received. The group recommended that most outcomes are measured at baseline and annually.

Conclusion The international minimum standard set of outcomes has the potential to improve clinical decision making through systematic measurement and comparability. This will be key in improving the standard of health care for people with PD across the world.
\end{abstract}

Keywords ICHOM $\cdot$ Patient-reported outcomes $\cdot$ Personality disorder $\cdot$ Quality of life $\cdot$ Risk-adjustment variables $\cdot$ Delphi procedure

Valentina Prevolnik Rupel

rupelv@ier.si

1 Institute for Economic Research, Kardeljeva ploščad 17, 1000 Ljubljana, Slovenia

2 International Consortium for Health Outcomes Measurement, Cambridge, USA

3 University of Amsterdam, Amsterdam, The Netherlands

4 Patient Representative, The Norwegian National Advisory Unit On Personality Psychiatry, Oslo, Norway

5 The Institute of Mental Health, WLMHT Managed Clinical Network, Southall, UK

6 Erasmus MC, Rotterdam, The Netherlands

7 Patient Representative, London, UK

8 The University of Melbourne, Melbourne, Australia

\footnotetext{
9 Patient representative, Te gek!?, Ghent, Belgium

10 Patient Representative, Ghent, Belgium

11 University of Toronto, Toronto, Canada

12 Auckland University of Technology, Auckland, New Zealand

13 University of Bristol, Bristol, UK

14 Tecnologico de Monterrey, Monterrey, Mexico

15 University of Houston, Houston, USA

16 Norwegian University of Science and Technology (NTNU), Trondheim, Norway

17 University of Central Lancashire, Preston, UK

18 Imperial College, London, UK

19 Swinburne University of Technology, Melbourne, Australia

20 Orygen, Melbourne, Australia
} 


\section{Introduction}

People with personality disorder (PD) have problems in functioning of aspects of self and interpersonal dysfunction which lead to emotional distress and impaired social function [1]. With onset early in life [2], high prevalence of over $5 \%$ of the general population and $50 \%$ in the outpatient psychiatric settings [3], it contributes to a substantial portion of health-care spending [4]. Most of the costs are incurred by inpatient and community mental health care and increased levels of unemployment and lost productivity among people with PD. A variety of psychological and psychosocial interventions have been shown to improve the mental health of people with PD [5-7]. The wide consensus is that the primary treatment for PD should be outpatient psychosocial therapy, with pharmacological treatment used mainly for the treatment of coexisting conditions. Further recommendation regarding the length and modality of treatments for each trait profile of PD is not clear, differs among countries and are often not in line with the latest research [8]. Compared to other common mental disorders, personality pathology is rarely tracked in routine clinical care. While many settings routinely assess the outcomes of people with depression and anxiety [9], outcome assessment in PD is rare and mostly refers to borderline personality disorders (BPD). In BPD, meta-analyses and reviews highlight the variety of outcomes utilized. Stoffers et al. [10] defined primary outcomes, which included overall BPD severity and BPD symptoms severity; and secondary outcomes, which included psychiatric comorbidity, general distress, global assessment of functioning, attrition/noncompliance with treatment, and adverse events. Lieb et al. [11] used all the outcomes from Stoffers et al. but added hospitalizations, emergency department visits, medication tolerability and side effects. Further outcomes measured in longer-term studies are social and vocational functioning, symptomatic remission and recovery from BPD [12].

In order to establish the value of each treatment for each service user, monitoring of health outcomes is essential. Value of treatment is defined as 'the outcomes achieved relative to the costs' [13]. For multiple reasons, measuring outcomes in the mental health is less common and more difficult than elsewhere, in spite of many available and validated health outcomes measures [14]. First of all, measurement precision of instruments might be lower compared to biomarkers. In addition, many instruments are time consuming, and clinicians might lack resources to implement them in busy clinical settings [14]. Also, there are many outcome measures available to measure each domain or symptom, making the results difficult to compare.

ICHOM was established to review the existing outcome measures that matter most to patients and to outline minimum standard sets of outcomes, measurement instruments, timepoints and risk adjustment factors for various conditions [15]. In 2018, ICHOM set out to cover some of the most prevalent mental health conditions. An international, multidisciplinary working group, led by ICHOM, was set up in the end of 2018. Our aim was to define the outcomes that matter most to persons with PD and prepare the standardized set of instruments to measure these outcomes.

\section{Methods}

\section{The working group}

The development of standard set for PD was initiated by ICHOM, which sets up a small project team (M.C., L.S.F., B.J., L.-M. C, T.G and V.P.R) and a wider working group. The wider working group consisted of 16 experts, including clinicians, nurses, patient representatives and experts in the area of outcome measurement. Working Group selection criteria defined by ICHOM were strictly followed; the members of the Working Group committed to active engagement and participation and were selected to cover the breadth of expertise needed to develop the content of the standard set-clinical expertise, PROMs expertise and health-care system evaluation expertise. The working group members came from Europe, North America, Latin America, Middle East, Australia and New Zealand, representing all regions of the world. Their work was coordinated and guided by the project team.

\section{Work process and decision making}

The working group convened via eight video calls from March 2019 to March 2020. Their work followed the Delphi process, previously modified and applied by ICHOM in the course of preparation of standard sets for a number of conditions [16-21]. A standard set of outcomes was developed through several phases (Fig. 1). Each teleconference had a previously determined goal, which was defined according to the issues that arose in the process of the development of the standard set. In line with the set goal, the project team prepared the research inputs based on the reviews of literature using common databases (PubMed, EMBASE, CINAHL, Medline, PsycINFO) and reviews of treatment guidelines and registries (e.g. Personality Disorders Registry Spain; Guideline on BPD: recognition and management, England; Guideline on Antisocial Personality Disorder: prevention and management, England; Guideline on Antisocial behaviour and conduct disorders in children and young persons: recognition and management, England; Guideline on Personality Disorders, Germany; National Outcomes 


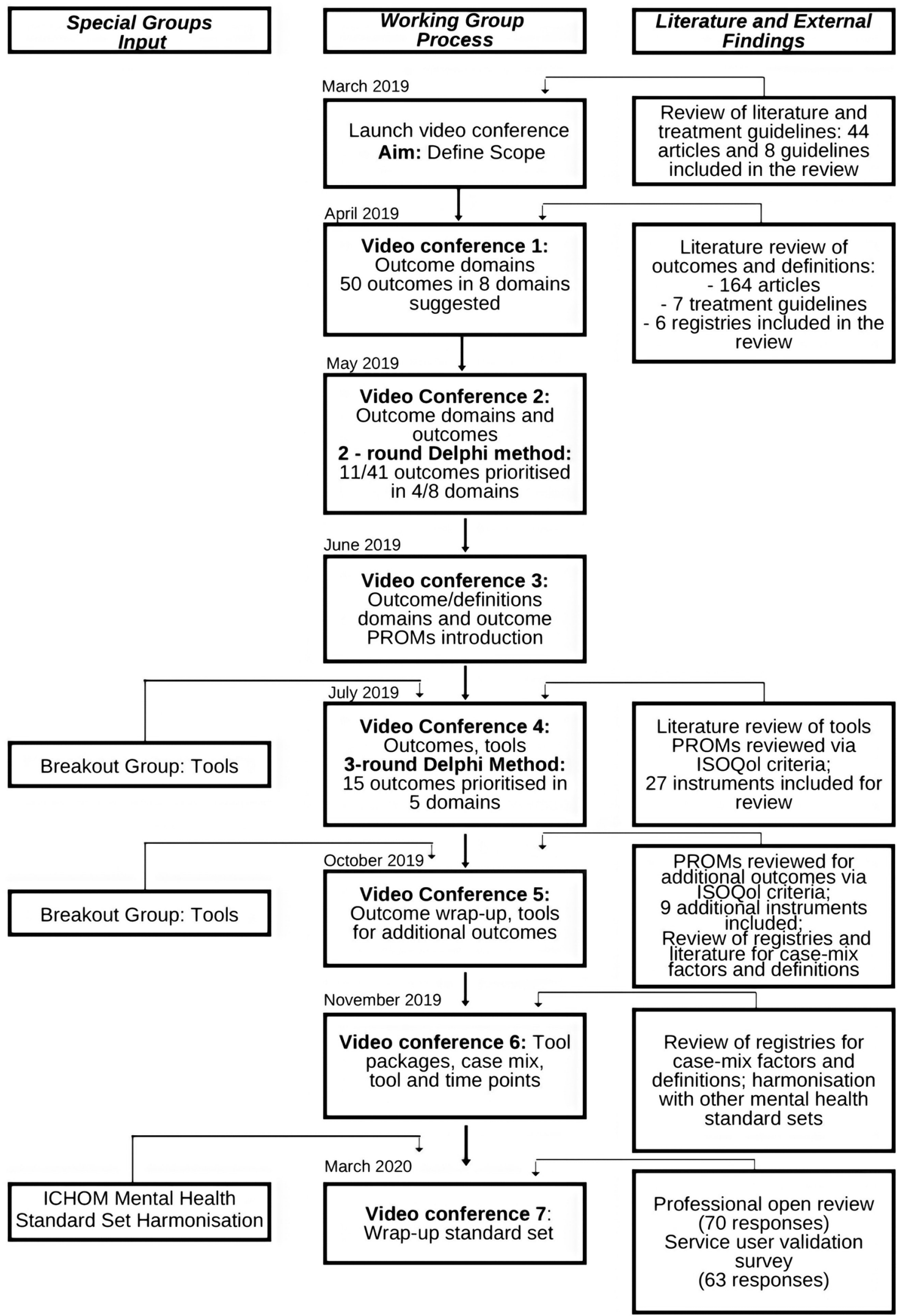

Fig. 1 Process of the outcomes development 
and Case-mix Collection (NOCC), Australia; APA's Mental health registry PsychPRO, USA; Mental Health Registry). Additionally, breakout groups were set up to discuss most relevant issues to decrease the complexity of the issues to be decided on at the working group calls.

Breakout groups were organized to discuss the issues of instrument selection and packages as well as to harmonize standard sets regarding outcome instruments, timepoints and case-mix variables across mental health working groups. At the teleconferences, gathered and analysed information, including proposals, was presented for group discussion. After each teleconference, the discussed content was organized into an online survey. It was emailed to working group members who were invited to vote on the issues discussed.

Content was included if $70 \%$ consensus was reached and excluded if less than $50 \%$ consensus was reached. Issues that remained inconclusive were further discussed and subjected to additional rounds of voting until a consensus was reached, following the rules from the previous sentence. At least an $80 \%$ of the group had to take part in a vote for it to be considered valid. A consensus had to be reached in four major decision areas: (1) scope: which conditions, population age and treatments should be included in the PD standard set, (2) outcome domains and outcomes in each domain, (3) instruments and instrument packages in each of the domains and (4) case-mix variables and timepoints.

To vote on outcomes, working group members discussed the long list of potentially relevant outcomes on the call and then voted online anonymously after reviewing the materials and minutes from the call. This was done using an online survey, where they were presented with each outcome and asked to rate the outcome on a scale from 1 to $9(1=$ not important, $9=$ essential $)$. Inclusion in the standard set required that a minimum of $80 \%$ of the consensus working group voted an item as "essential" (score of 7-9) in the first or second round Delphi vote. When consensus was not reached by voting, the item was discussed and revisited in the next videoconference and survey. Outcomes were excluded if a minimum of $80 \%$ of the consensus working group voted an item as "not recommended" (score 1-3). The consensus working group voted on all inconclusive outcomes in the final survey round, following ICHOM processes, in which the response options were simply "include" or "exclude". In this final round, inclusion in the standard set required only $70 \%$ consensus. A similar process was used to reach consensus on recommended measures and risk adjustment factors.

\section{Definition of scope and selection of outcome domains and outcomes}

Preceding the launch call, a systematic literature review was performed in November 2018 to define the scope of the work.
The following databases were searched: Medline and Embase in Ovid and CINAHL and Psychinfo in Ebsco. Out of 3270 articles identified, 49 were included in the scope definition. Due to the high number of hits the decision was taken to conduct all further searches in Medline at first and only extend the search to other databases if necessary. The following systematic literature search for outcome domains was conducted in Medline in March 2019 (Fig. 2). Additionally, treatment guidelines and registries were taken into account to develop the final definition of outcome domains and outcomes.

\section{Selection of outcome measures}

The selection of outcome measures was based on the systematic literature review in Fig. 2. A total of 268 potentially relevant patient-reported outcome measures (PROMs) were screened with respect to (1) conceptual and measurement model, (2) evidence supporting psychometric properties, e.g. validity and reliability, (3) clinical utility, (4) feasibility of implementation (licensing fees - measures that need to be paid for were excluded, number of language translations, number of citations, and service user and administrative burden - length of the questionnaire and (5) harmonization with other mental health standard sets. Additional literature searches were conducted in PubMed for each measure undergoing screening. The measures that passed the initial screening by the project team of the 268 potentially relevant patient-reported outcome measures (PROMs) identified were then presented to the working group, alongside evidence supporting psychometric properties, e.g. validity and reliability. The working group discussed the issues around clinical utility, psychometric properties, feasibility of implementation and benchmarking potential during the working group call.

Following this discussion, the working group members voted anonymously on an online survey about which measure should capture which outcome individually. The decision to include or exclude a measure required $70 \%$ consensus, with a minimum of $80 \%$ participation from working group members.

To establish cross-cultural equivalence between the various countries, a list of case-mix variables was extracted from the registries and PD guidelines. Case-mix variables (Table 4) describe the context in which the outcomes are measured. To ensure high level of harmonization, previous ICHOM standard sets were reviewed for definition of demographic and socioeconomic variables.

\section{External validation by health professional and service user experts}

In February 2020, ICHOM presented a draft recommended PD standard set, which was sent into open review process by 


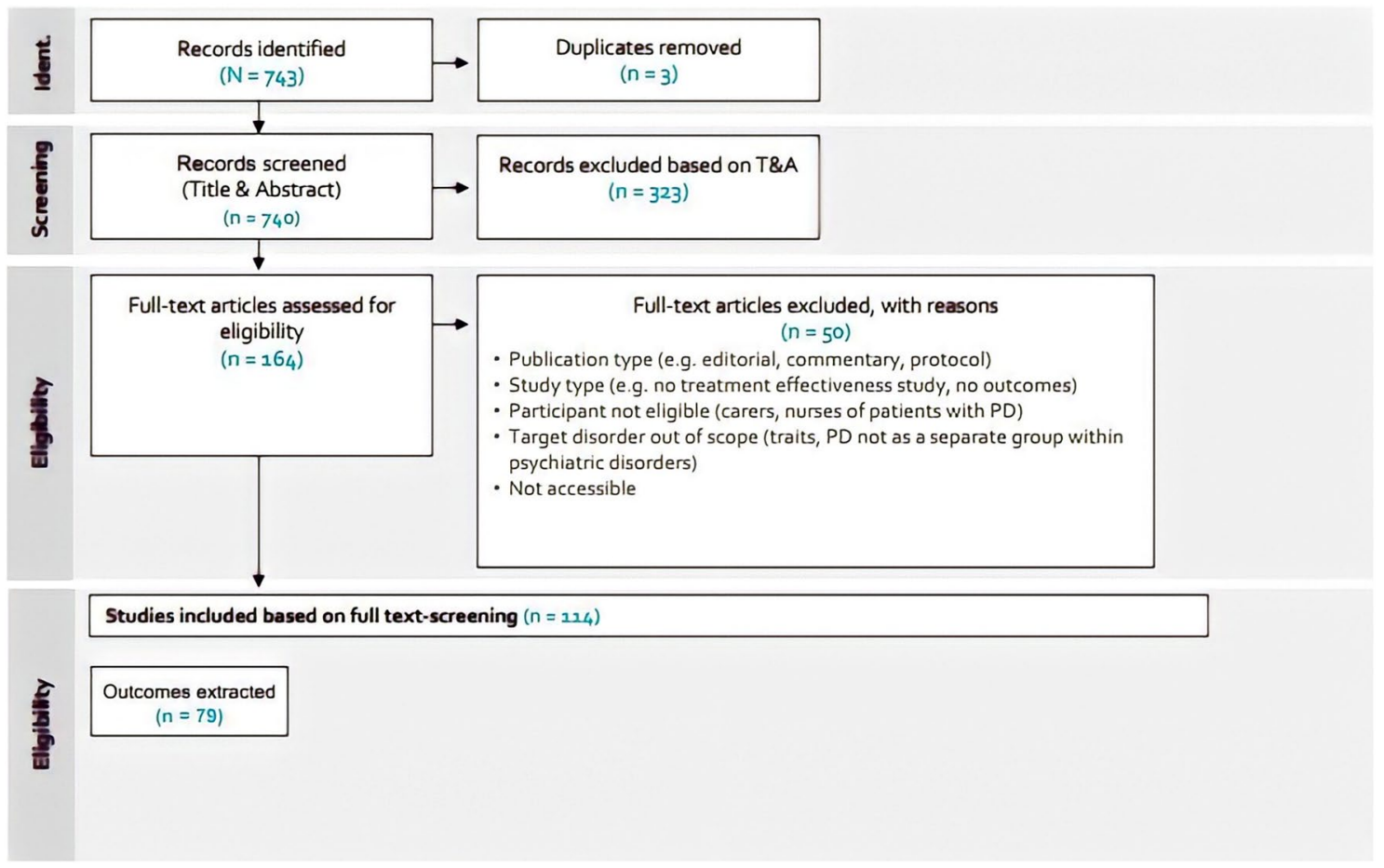

Fig. 2 Search strategy and selection process for final inclusion of outcome domains considered for the final PD standard set

professionals and into service user validation process. Any results securing an endorsement higher than $70 \%$ from the open review panel (service users) were accepted, while those receiving a lower endorsement went into further discussion with working group members.

Search term: ("personality disorder" [ti] OR "borderline personality disorder"[tiab] OR "schizotypal personality disorder"[tiab] OR "schizoid personality disorder"[tiab] OR "histrionic personality disorder" [tiab] OR "narcissistic personality disorder"[tiab] OR "paranoid personality disorder"[tiab] OR “avoidant personality disorder"[tiab] OR "antisocial personality disorder"[tiab] OR "dependent personality disorder" [tiab] OR "obsessive-compulsive personality disorder" [tiab] OR "Negative affectivity in personality disorder or personality difficulty" [tiab] OR "Detachment in personality disorder or personality difficulty" [tiab] OR "Dissociality in personality disorder or personality difficulty"[tiab] OR "Disinhibition in personality disorder or personality difficulty"[tiab] OR "Anankastia in personality disorder or personality difficulty"[tiab] OR "Borderline pattern"[tiab]) AND (meta-analysis [ti] OR review [ti]). Articles from 2009 on were included.

\section{Results}

\section{Scope}

The working group decided to include PD as defined by International Classification of Diseases 11 th revision (ICD-11) [1]. Substance use-induced PD, PD due to organic causes including head injury, personality change/ disorder secondary to other mental health condition, subthreshold personality dysfunction and personality difficulty were excluded from the scope of the project. The settings included primary care, inpatient and outpatient care, day hospital, community treatment, forensic mental health services, family care, and criminal justice care in a form of group as well as individual therapies. All psychotherapeutic and pharmacological treatments were voted within scope, except use of drugs for comorbid conditions. Recommendations were limited to adults and adolescents aged 13 years or above - for children aged 2-12 that there is not much literature on PDs and the outcomes measures used are different. The literature [22] suggests 
that PD begins in childhood and adolescence, and can be diagnosed in young people. For example, BPD is common among young people: the estimated prevalence is $1-3 \%$ in the community, rising to $11-22 \%$ in outpatients, and $33-49 \%$ in inpatients. BPD is one of the leading causes of disability-adjusted life years (DALYs) in young people among mental diseases and represents a substantial financial burden for the families of young people. The effectiveness of structured treatments for BPD in young people has been demonstrated.

\section{Outcome domains and measures}

Based on the literature review, a list of 50 outcomes in eight outcome domains was proposed for voting. This list was later expanded and refined following the suggestions, discussion and three rounds of Delphi voting by working group members. The final list consists of 14 outcomes, grouped in four outcome domains [9]: (1) Mental health, (2) Behaviour, (3) Functioning and (4) Recovery. All the outcomes considered for the inclusion in the PD standard set are presented in Table 1.

A comprehensive literature review was performed for each of the outcomes in order to identify the instruments within the defined scope of the standard set. A total of 268 instruments identified were screened and reduced to 13 instruments (Table 2). A breakout group was established to help ensure that the measures were harmonized to the highest possible degree among mental health standard sets. As there were four mental health sets in development simultaneously and all of them included "Functioning" and "Health-Related Quality of Life", the same instruments to cover the same domain across the mental health sets were used. Members of the group also expressed a preference for measures that were appropriate for both adolescents and adults in order to enable tracking the mental health outcomes during this period.

As the number of the outcomes was high, measures that could cover more than one domain were looked for, which could later be complemented by additional instruments. Measures with positive framing of the questions were preferred: this decision was made by the working group following feedback on the content and phrasing from the lived experience representatives.

Due to a high degree of overlap in the domains that different measures covered, instrument package options were then prepared for voting in the final phase. The group aimed to ensure that the final package of measures would take a person less than $25 \mathrm{~min}$ to complete. The final outcomes and the measures are presented in Fig. 3 and Table 3. While most of the outcomes are core, "Emotional Dysregulation", "Aggression" and "Self-Harm" were included as additional outcome measures for use only in those who experience them. No adequate instrument for "Coping with Past Experiences of Trauma" was identified.

Table 1 List of all outcomes proposed for voting to working group

\begin{tabular}{lll}
\hline Included outcomes & Excluded outcomes & Mental well-being \\
\cline { 2 - 3 } Emotional dysregulation & Anxiety & Community participation \\
\hline Emotional distress & Capacity for empathy & Resilience \\
Suicide ideation and behaviour & Depression/low mood & Self-compassion \\
Self-harm & Dissociation & Self-esteem \\
Impulsivity & Emptiness & Self-efficacy \\
Global functioning & Guilt & Caregiver-youth relationship \\
Interpersonal functioning & Hopelessness & Criminal activity \\
Social functioning & Obsessive rigidity & Family burden \\
Sense of belonging & Personality organization/pathology/temperament & Family mental health \\
Self-care & Suicide & Stigma \\
Health-related quality of life & Substance misuse & Time to treatment \\
Identity disturbance & Abuse of others (harming loved ones) & Time to diagnosis \\
Aggression & Pain & Use of health services \\
Severity of personality disorder & Mortality & Hospital admission \\
Coping with Past Experiences of Trauma* & Sleep & Cost of treatment and care \\
& Self-awareness & Use of other services \\
& Sense of hope & Patient-reported experience \\
\hline
\end{tabular}

*No adequate instruments for 'Coping with Past Experiences of Trauma' were identified 


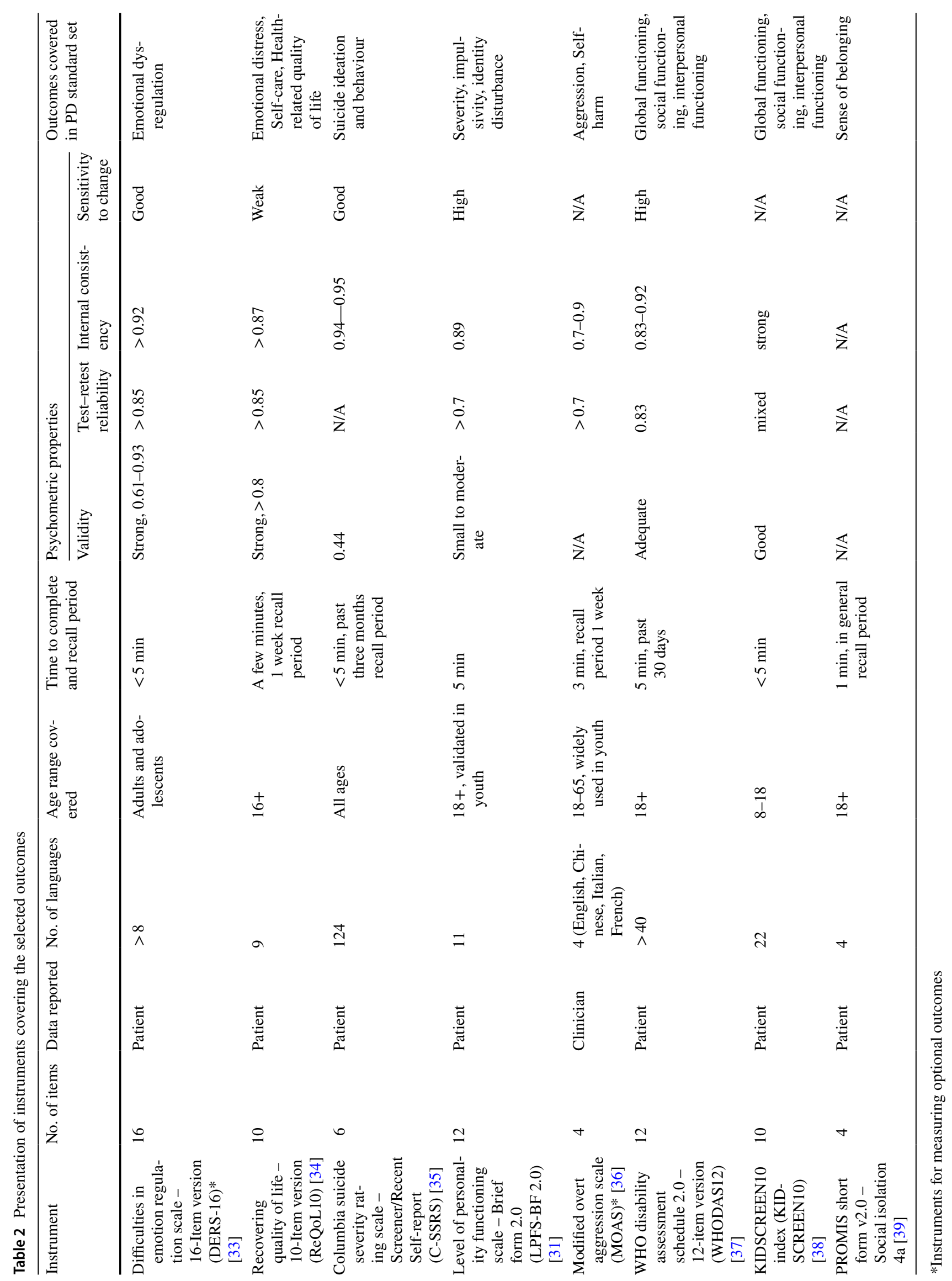


ICHOM Personality Disorders Standard Set - Tool package

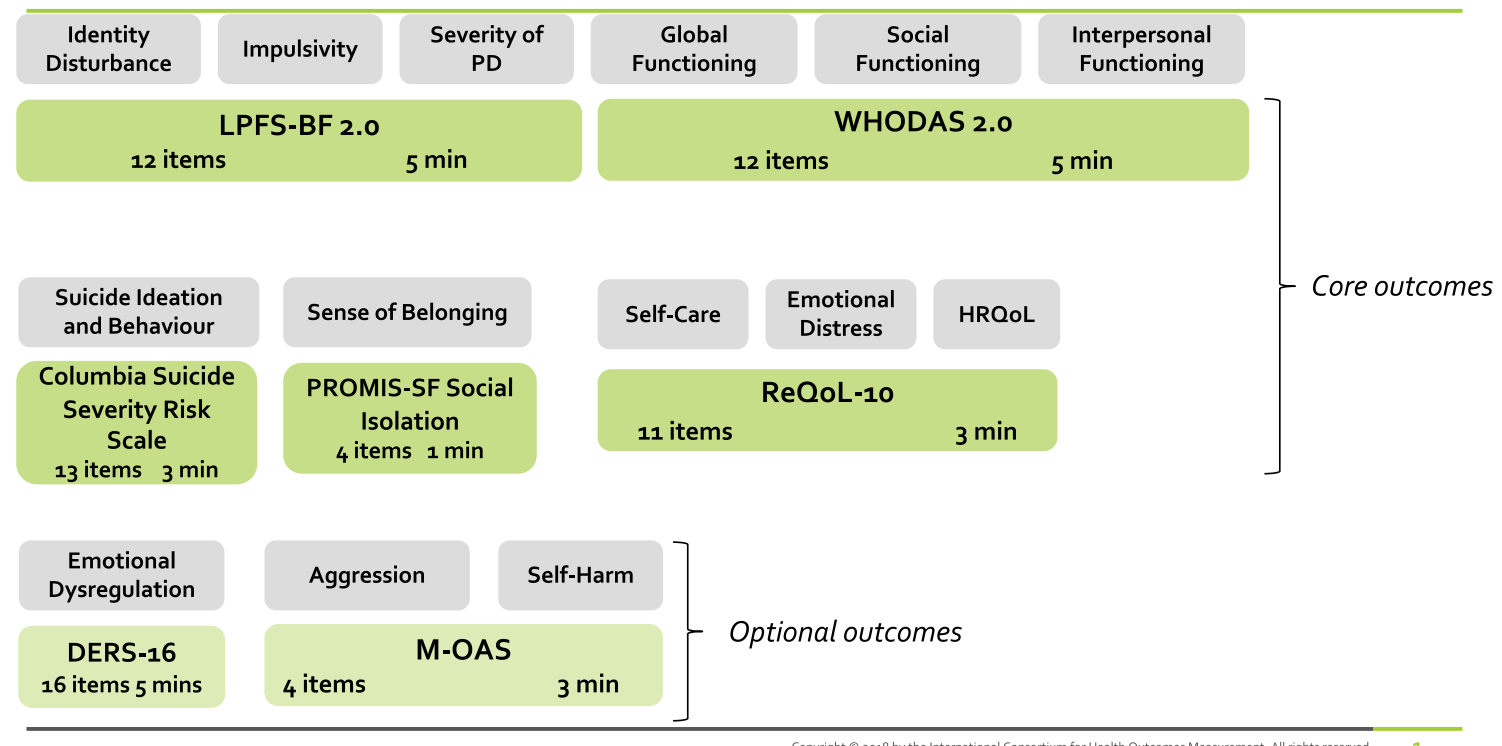

Fig. 3 Recommended instrument package with assigned outcomes coverage and timing

\section{Case-mix variables}

Case-mix variables are included in the standard set in order to ensure the baseline comparability of treatment populations and intervention factors. ICHOM seeks to extract a minimum set of case-mix variables. Initially, a literature review and extraction from the registries and PD guidelines were performed to identify possible case-mix variables. Case-mix variables were compared against the other ICHOM mental health standard sets, and a harmonized version consisting of demographic and intervention factors was confirmed by the working group (Table 4).

\section{Data collection timepoints}

Recommended timepoints for the collection of data should be looked at as the minimum requirement for measuring the defined outcomes. The outcome assessment timeline was proposed by the working group to best achieve a balance between the clinically relevant times when outcomes may be expected to change, and the pragmatic concerns in data collection. To harmonize across ICHOM mental health Standard Sets, a meeting between ICHOM mental health working group chairs was held to discuss the timepoints recommendation and suggestions were later voted on by each working group independently. The consensus reached was to recommend assessing outcomes prior to treatment as a baseline, every 3 months in continuous treatment until the discharge and then 6 months after discharge and annually thereafter when not in continuous treatment (Fig. 4).

\section{Validation process}

Seventy responses were received from mental health professionals in 17 countries. The survey was conducted online anonymously, and the respondents used a link to access and complete the survey. The survey was published on ICHOM's website and shared within a number of newsletters, the mailing lists of which were not disclosed to the authors of this manuscript. No further variables were collected from the respondents. All outcome domains included in the initial recommendation received high endorsement (85\% confidence in overall domains) by the professionals in the open review panel. Sixty-three service users responded to the questions in the service users validation survey and the outcomes 'Aggression', 'Identity Disturbance' and 'Emotional Dysregulation' did not reach 70\% endorsement. However, these outcomes, as well as the measures proposed to capture them, were highly endorsed by the professional open review panel. All three outcomes were discussed with the working group again, and the proposal was formed to include all the outcomes in the standard set. However, it was decided that 'Emotional Dysregulation' and 'Aggression' would not be part of the core list of outcomes; the rationale being that not all people with PD experience aggression and emotional dysregulation. 


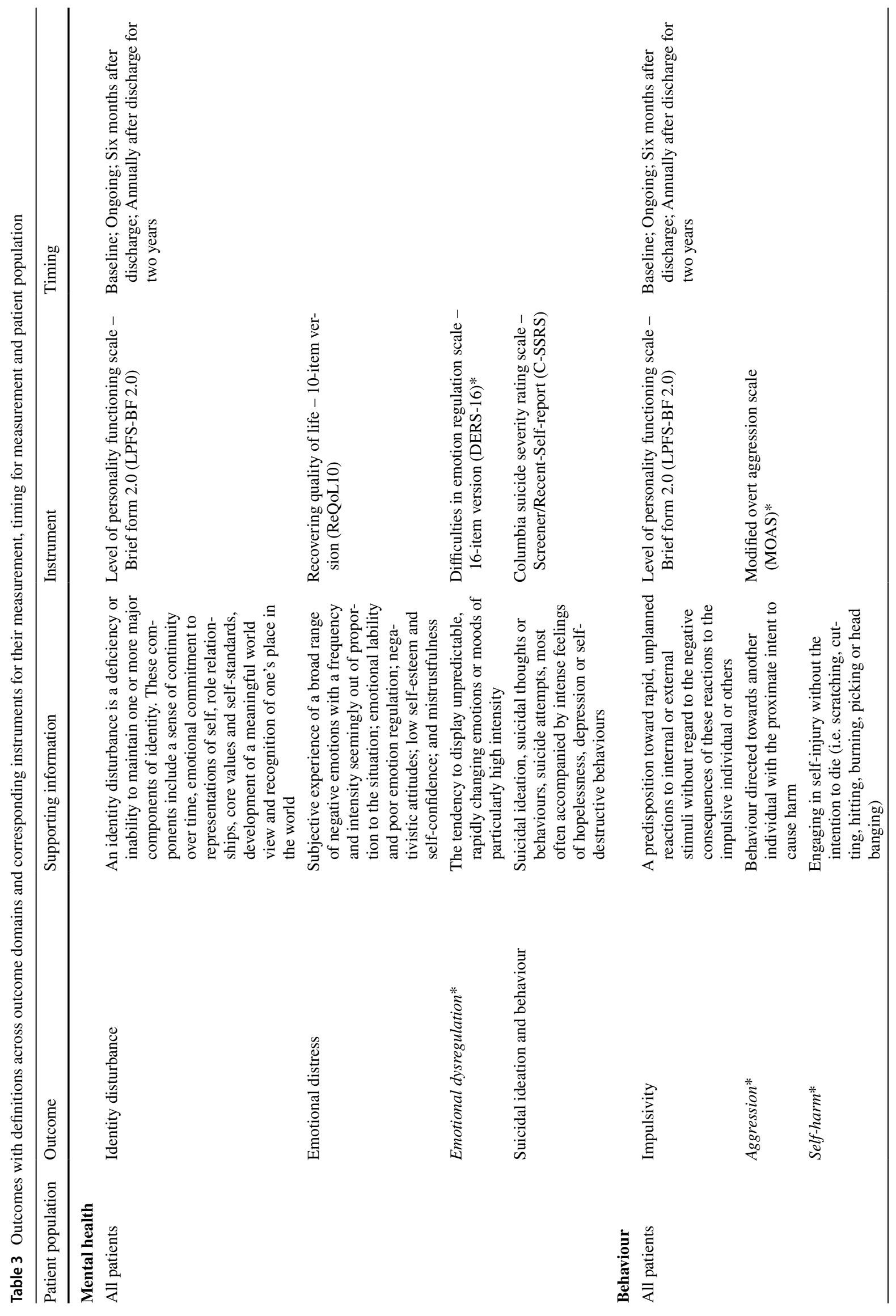




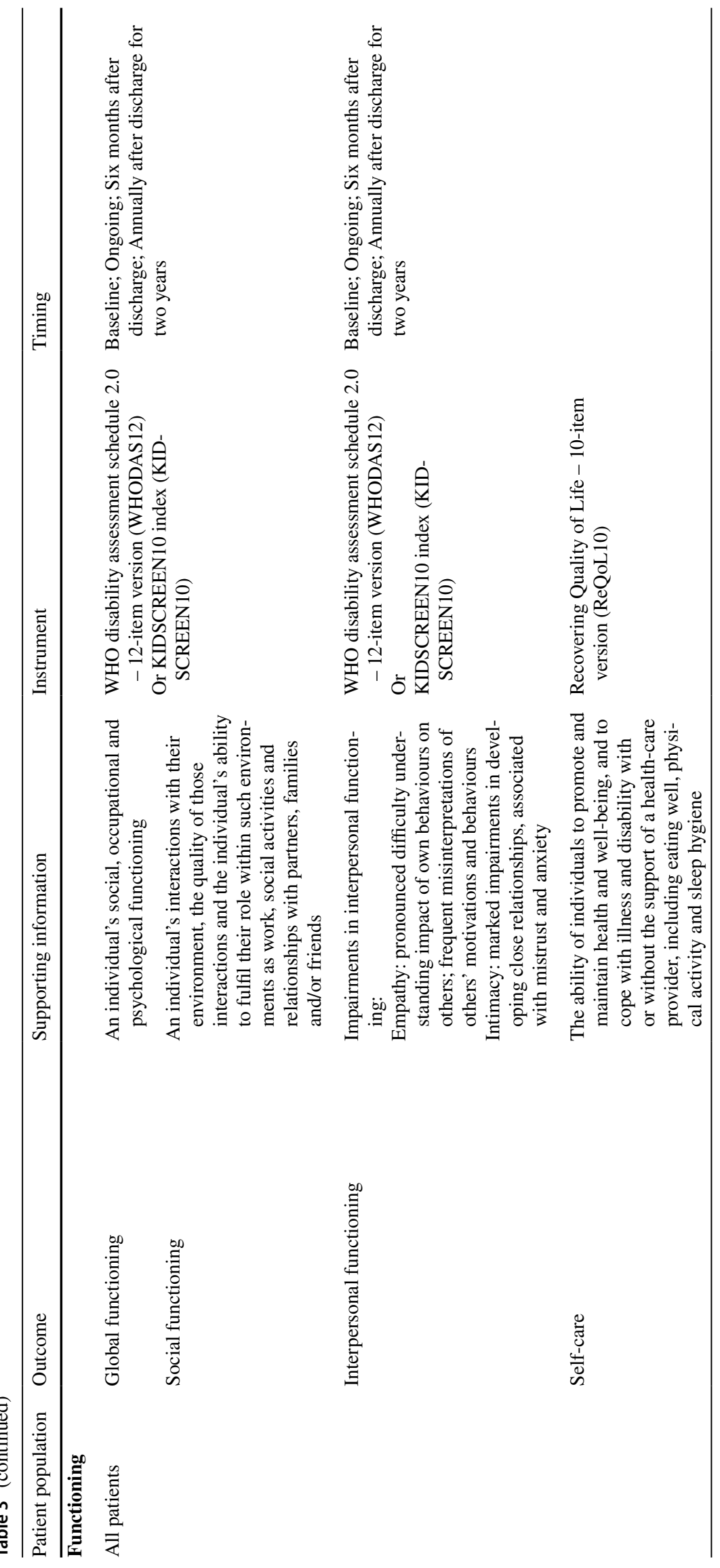

Springer 


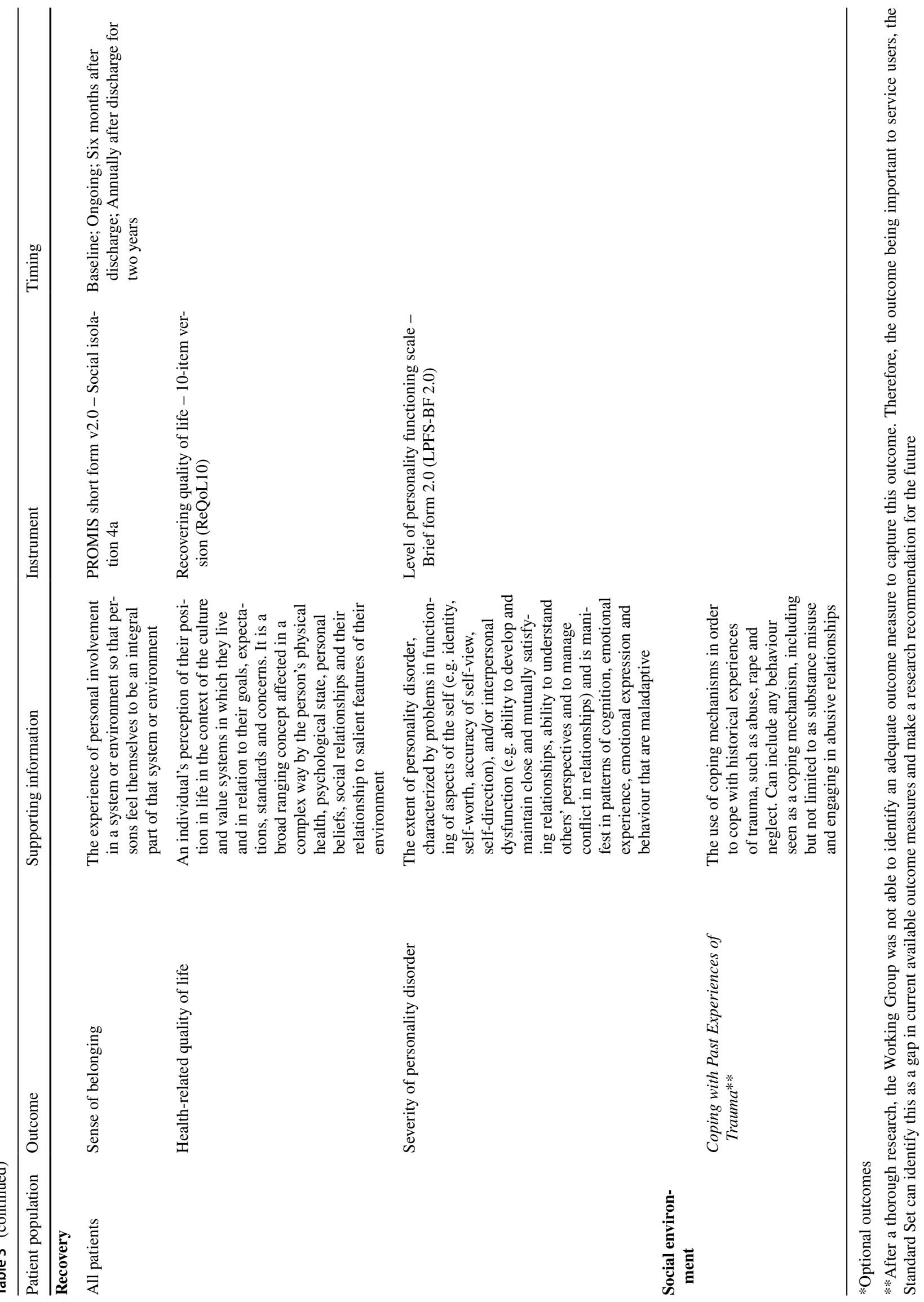




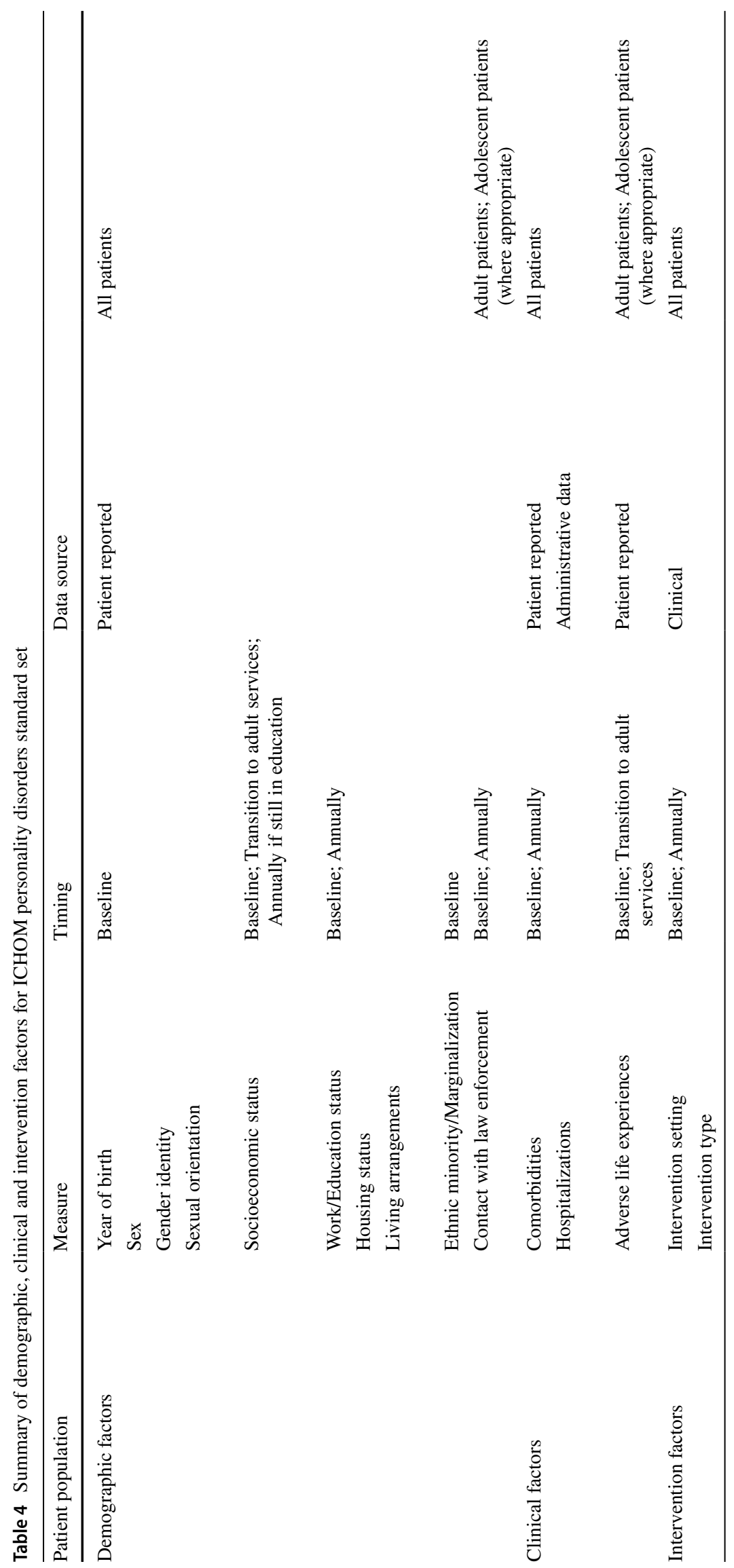




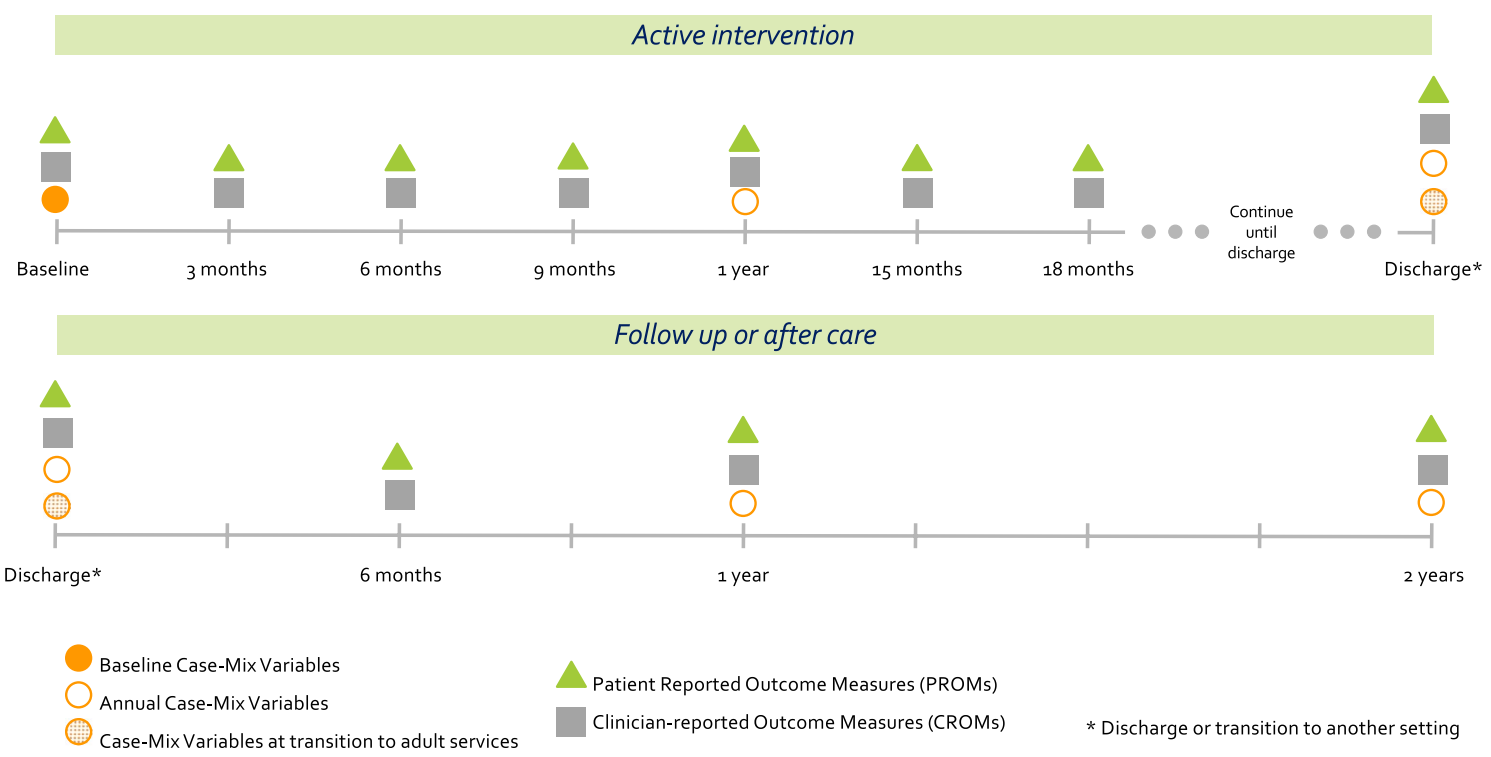

Fig. 4 Time guidance on the variables collected from service users and clinicians

\section{Discussion}

As the case for measuring patient outcomes becomes increasingly accepted by clinicians and decision makers in health care, one of the challenges we are faced with is selecting which measures to use from among the vast array of different instruments that could be used. The ICHOM working group for PD responded to this challenge by selecting and defining a standardized minimum set of outcome measures that would be appropriate to use across different cultural and geographical settings [23]. The included outcomes represent those that matter most to people with PD. The measurement of these outcomes across different environments should help to build better communication between patients and providers. Benchmarking of the results should motivate and empower providers to seek and share good practices and improve care and clinical protocols; payers would be able to clearly see the value of care and make informed decisions on strategic purchase strategies [24]. All the outcomes alongside casemix variables, timepoints for collection and questionnaires are freely available at the ICHOM website (https://www. ichom.org/standard-sets/).

ICHOM entered the mental health area in 2018. This area depends to an even higher degree on patient-reported outcomes in comparison with some other clinical areas, where clinical readings can describe the outcomes relatively better. Previous research [25] has shown that defining patientreported outcomes for PD, particularly BPD has many challenges. BPD has heterogeneous clinical features, meaning that patient-reported outcomes should include broad assessment of psychopathology, but at the same time, measure stable as well as more dynamic aspects of the disorder. Social and occupational functioning are especially salient when assessing the outcomes of people with PD, because a number of studies have shown impaired functioning even when mental health improves [26]. Crawford et al. [27] conducted a Delphi study with service providers, services users and academic experts and similarly established that people with a wide range of PD felt that the most important outcome measure that should be assessed was healthrelated quality of life, followed by mental health and social functioning. Previous attempt [12] to identify core outcome measures in PD that capture quality of life, functioning and symptoms, highlighted that the number of outcomes for BPD is extensive. Above all, this attempt as well as the guidelines on the development of an agreed set of outcomes measures [26] were focused solely on BPD, while the ICHOM recommended standard set is designed for all those with PD and related mental health conditions.

During the whole working process, lasting between October 2018 and June 2020, many scientists, clinicians and service user representatives were included in the formulation of the standard set. All the members discussed the different steps of the work, from defining the scope to the preparation of the final manuscript. Due to the long period of preparing the final set, there was quite a high degree of fluctuation in the project team, as well as in the working group, but all involved expressed their valuable opinion and contributed effectively to the final outcome. The inclusion of the working group members was, however, based on their work recommendations and limited to people from 17 countries. In spite of extensive literature reviews and use of Delphi processes throughout the 
project, the results might have differed with a different group of participants from different cultural backgrounds.

The primary aim of the standard set is to reflect outcomes that are important to service users. Therefore, including their views and extensive inputs through the whole process is a strength of this work. There were six service user representatives included in the working group and, in the end, 63 users (among them seven carers or parents) reviewed the final version of the standard set, with $94 \%$ saying that all important outcomes are captured in the standard set and $96 \%$ saying that it would be useful having these outcomes collected. All of the service user representatives came from developed countries and most of them are from Europe.

The process of data collection via suggested questionnaires represents a significant time burden for service users as well as for clinicians. The participants had this in mind and tried to cover all the outcomes in the standard set with as few instruments as possible. Still, the outcomes in the final standard set are measured by eight instruments and the complete collection of all outcomes lasts up to $30 \mathrm{~min}$ (including optional instruments). In many countries, the collection of PROMs is still not supported by information communication technology that would enable more efficient collection of data, less reluctance of the stakeholders and automated analysis and results. ICHOM is working on the information of data collection to support the users of the standard sets. In order to promote the use of the standard set in all international environments, the selected outcomes we chose are already translated into many languages. They are available in multiple formats, easily integrated into diverse data collection tools, are computer adaptive and can be used free of charge. A very important issue in mental health standard sets is comorbidity of PDs with other mental health disorders, such as substance use disorders [28], attention-deficit hyperactivity disorder [29] and schizophrenia [30]. Therefore, harmonization of measures across the mental health standard set, as well as among other standard sets that include the same domains, is an important issue, which was taken into account in the process of the selection of measures.

Available evidence suggests that self-assessed measures made by people with PD have high test-retest reliability [31]. However, concerns have been raised about the reliability of self-reported accounts of aggression and other externalizing behaviours [32]. ICHOM aims to establish person-centred outcomes. However, in recognition of the challenges of relying on self-report measures of aggression, a clinician-rated measure, the Modified Overt Aggression Scale, was selected to measure this outcome.

After undertaking a thorough systematic review, the working group was not able to identify an adequate outcome measure to capture the "Coping with Past Experiences of
Trauma' outcome. As the outcome is important to service users, the working group identified the lack of an appropriate instrument as a gap in the currently available outcome measures. Future research should be directed toward defining an appropriate instrument to measure this outcome. Various scales measuring similar constructs, such as 'Posttraumatic growth' have been looked at in the process and some of them overlap with 'Coping with Past Experiences of Trauma'. As such, they might be helpful in defining an appropriate measure in the future. Additionally, discriminatory effects among different types of PD through the Standard Set of this ICHOM endeavour need to be studied further.

Furthermore, the standard set is not seen as fixed but should be updated regularly, following new developments in the clinical environment, as well as developments in the health measurement area. The standard set should be seen as a minimal set of outcomes and instruments for their measurement, and further outcomes and measures could be freely added to this set if needed.

\section{Conclusions}

The development of a minimal standard set of value-based service user-centred outcome measures in PD should lead to higher value of care, and better outcomes of care, for people with PD all across the world. Widespread use of these measures will lead to benchmarking and exchange of good practices, to greater inclusion of service users in care processes, and to better communication between clinicians and service users. It will also provide the payer with evidence that could serve as a basis for informed decision making on allocation of funds.

Acknowledgements We would like to thank all external stakeholders for the time and effort contributed without financial compensation. We also extend our gratitude to Doctor Alireza Farnam and Professor Martin Bohus, who contributed to working group discussions. This work represents the views of the working group members; no representation of the views of their respective institutions is implied. Paul Moran is supported by the NIHR Biomedical Research Centre at University Hospitals Bristol and Weston NHS Foundation Trust and the University of Bristol.

Funding This project was made possible thanks to by the generous contributions of NHS England and NHS Improvement; the NSW Agency for Clinical Innovation, Australia; Providence Health Care, USA; and Region Västra Götaland, Sweden. Members of the working group did not receive financial compensation for their participation. The opinions expressed in this article are those of the authors; no representation of the views of the funding sources is implied. The funders played no role in the study design, collection, analysis or interpretation of the data, writing of the report, or the decision to submit the article for publication. 


\section{Declarations}

Conflict of interest Anroud Arntz, Julia Blazdell, Lisa-Marie Chadderton, Charlotte Delvaux, Timea Gintner, Beth Jagger, Lucie Langford, Brian McKenna, Karla Patricia Pacheco Alvarado, Carla Sharp, Luz Sousa Fialho, Fieke van Gorp and Karen M. Wright declare no conflict of interest. Åse-Line Baltzersen works as Communication advisor for The Norwegian National Advisory Unit on Personality Psychiatry. Marika Cencelli works for Mental Health Policy and Strategy, NHS England and NHS Improvement. Andrew Chanen received grants from National Health and Medical Research Council and Australian Research Council, is a Director of Clinical Programs and Services, Orygen, Melbourne, Australia, Professorial Fellow, Centre for Youth Mental Health, The University of Melbourne and Board member of National Education Alliance for Borderline Personality Disorder (NEABPD) Australia. Mike Crawford received professional fees from Central \& North West London HHS Foundation Trust and grant from National Institute for Health Research and the Department of Health on behalf of Imperial College London; is a Professor of Mental Health Research, Imperial College London, Director of the College Centre for Quality Improvement at the Royal College of Psychiatrists and Trustee of the Maytree (a charity providing support for people in a suicidal crisis). Paul Moran received grants from the National Institute for Health Research and the Department of Health; The Above \& Beyond Charity. Valentina Prevolnik Rupel and Jan van Busschbach are members of EuroQol Group. Wei Wang received grant from Zhejiang University and Natural Science Foundation of China.

Open Access This article is licensed under a Creative Commons Attribution 4.0 International License, which permits use, sharing, adaptation, distribution and reproduction in any medium or format, as long as you give appropriate credit to the original author(s) and the source, provide a link to the Creative Commons licence, and indicate if changes were made. The images or other third party material in this article are included in the article's Creative Commons licence, unless indicated otherwise in a credit line to the material. If material is not included in the article's Creative Commons licence and your intended use is not permitted by statutory regulation or exceeds the permitted use, you will need to obtain permission directly from the copyright holder. To view a copy of this licence, visit http://creativecommons.org/licenses/by/4.0/.

\section{References}

1. WHO. (2019). International classification of diseases 11th revision. World Health Organization. Retrieved 13 July 2020 from https://www.who.int/classifications/icd/en/

2. Chanen, A., Sharp, C., Hoffman, P., \& Disorder, G. (2017). Prevention and early intervention for borderline personality disorder: A novel public health priority. World Psychiatry, 16(2), 215-216.

3. Winsper, C., Bilgin, A., Thompson, A., Marwaha, S., Chanen, A., Singh, S., et al. (2020). The prevalence of personality disorders in the community: A global systematic review and meta-analysis. British Journal of Psychiatry, 216(2), 69-78.

4. Rendu, A., Moran, P., Patel, A., Knapp, M., \& Mann, A. (2002). Economic impact of personality disorders in UK primary care attenders. British Journal of Psychiatry, 181, 62-66.

5. Doering, S., Hörz, S., Rentrop, M., Fischer-Kern, M., Schuster, P., Benecke, C., et al. (2010). Transference-focused psychotherapy v. treatment by community psychotherapists for borderline personality disorder: randomised controlled trial. British Journal of Psychiatry, 196(5), 389-395.

6. Vogt, K. S., \& Norman, P. (2019). Is mentalization-based therapy effective in treating the symptoms of borderline personality disorder? A systematic review. Psychology and Psychotherapy, 92(4), 441-464.

7. Storebø, O.J., Stoffers-Winterling, J.M., Völlm, B.A., Kongerslev, M.T., Mattivi, J.T., Jørgensen, M.S., et.al. (2020). Psychological therapies for people with borderline personality disorder. Cochrane Database of Systematic Reviews, 5, CD012955. https://doi.org/10.1002/14651858.CD012955.pub2

8. Simonsen, S., Bateman, A., Bohus, M., Dalewijk, H.J., Doering, S., Kaera, A. et al. (2019). European guidelines for personality disorders: Past, present and future. Borderline Personality Disorder Emotion Dysregulation 6, 9

9. Kendrick, T., El-Gohary, M., Stuart, B., Gilbody, S., Churchill, R., Aiken, L., et al. (2016). Routine use of patient reported outcome measures (PROMs) for improving treatment of common mental health disorders in adults. Cochrane Database System Review. https://doi.org/10.1002/14651858.CD011119.pub2

10. Stoffers, J.M., Völlm, B.A., Rücker, G., Timmer, A., Huband, N., Lieb, K. (2012). Psychological therapies for people with borderline personality disorder.Cochrane Database System Review, 15(8), CD005652.

11. Lieb, K., Völlm, B., Rücker, G., Timmer, A., \& Stoffers, J. M. (2010). Pharmacotherapy for borderline personality disorder: Cochrane systematic review of randomised trials. British Journal of Psychiatry, 196(1), 4-12.

12. Madan A, Fowler JC. (2015). Consistency and coherence in treatment outcome measures for borderline personality disorder. Borderline Personality Disorder Emotion Dysregulation. https:// doi.org/10.1186/s40479-014-0022-5

13. Porter, M. E. (2010). What is value in health care? New England Journal of Medicine, 363(26), 2477-2481.

14. Rose, M., \& Bezjak, A. (2009). Logistics of collecting patientreported outcomes (PROs) in clinical practice: An overview and practical examples. Quality of Life Research, 18(1), 125-136.

15. Porter, M. E., Larsson, S., \& Lee, T. H. (2016). Standardizing patient outcomes measurement. New England Journal of Medicine, 374(6), 504-506.

16. Martin, N. E., Massey, L., Stowell, C., Bangma, C., Briganti, A., Bill-Axelson, A., et al. (2015). Defining a standard set of patient-centered outcomes for men with localized prostate cancer. European Urology, 67, 460-467.

17. Allori, A. C., Kelley, T., Meara, J. G., Albert, A., Bonanthaya, K., Chapman, K., et al. (2017). A standard set of outcomes measures for the comprehensive appraisal of cleft care. Cleft Palate-Craniofacial Journal, 54, 540-554.

18. Vashaar, M.A.H.O., Gupta, Z.D., Bijlsma, J.W.J., Boonen, A., Chau, J., Couvoisier, D.S. et al. (2019). The international consortium for health outcomes measurement (ICHOM) set of outcomes that matter to people living with inflammatory arthritis: Consensus from an international working group. Arthritis Care and Research (Hoboken),71(12), 1556-1565.

19. Obbarius, A., van Maasakkers, L., Baer, L., Clark, D. M., Crocker, A. G., de Beurs, E., et al. (2017). Standardization of health outcomes assessment for depression and anxiety: Recommendations from the ICHOM depression and anxiety working group. Quality of Life Research, 26, 3211-3225.

20. Ong, W.L.O., Schouwenburg, M.G., van Bommel, A.C.M., Stowell, C., Allison, K.H., Benn, K.E. et al. (2016). A standard set of value-based patient-centered outcomes for breast cancer. The International Consortium for Health Outcomes Measurement (ICHOM) Initiative. JAMA Oncology, 3(5), 677-685.

21. Nijagal, M. A., Wissig, S., Stowell, C., Olson, E., Amer-Wahlin, I., Bonsel, G., et al. (2018). Standardized outcomes measures for pregnancy and childbirth, an ICHOM proposal. BMC Health Services Research, 18, 953.

22. Chanen, A. M., Sharp, C., Hoffman, P., \& The Global Alliance for Prevention and Early Intervention for Borderline Personality 
Disorder. (2017). Prevention and early intervention for borderline personality disorder: A novel public health priority. World Psychiatry, 16(2), 215-216.

23. Berenson, R. A., \& Kaye, D. R. (2013). Grading a physician's value-the misapplication of performance measurement. New England Journal of Medicine, 369(22), 2079-2081.

24. Dias, A. G., Roberts, C. J., Lippa, J., Arora, J., Lundstrom, M., Rolfson, O., et al. (2017). Benchmarking outcomes that matter most to patients: The GLOBE programme. EMJ, 2(2), 42-49.

25. Hasler, G., Hopwood, C. J., Jacob, G. A., Brändle, L. S., \& Schulte-Vels, T. (2014). Patient-reported outcomes in borderline personality disorder. Dialogues in Clinical Neuroscience, 16(2), 255-266.

26. Newton-Howes, G., Clark, L., \& Chanen, A. (2015). Personality disorder across the life course. Lancet, 385(9969), 727-734. https://doi.org/10.1016/s0140-6736(14)61283-6

27. Crawford, M. J., Price, K., Rutter, D., Moran, P., Tyrer, P., Bateman, A., et al. (2008). Dedicated community-based services for adults with personality disorder: Delphi study. British Journal of Psychiatry, 193, 342-343.

28. Parmar, A., \& Kaloiya, G. (2018). Comorbidity of personality disorder among substance use disorder patients: A narrative review. Indian Journal of Psychological Medicine, 40(6), 517-527.

29. Matthies, S. D., \& Philipsen, A. (2014). Common ground in attention deficit hyperactivity disorder (ADHD) and borderline personality disorder (BPD)-review of recent findings. Borderline Personality Disorder and Emotion Dysregulation, 1, 3.

30. Wei, Y., Zhang, T., Chow, A., Tang, Y., Xu, L., Dai, Y., et al. (2016). Co-morbidity of personality disorder in schizophrenia among psychiatric outpatients in China: Data from epidemiologic survey in a clinical population. BMC Psychiatry, 16, 224.

31. Hutsebaut, J., Feenstra, D. J., \& Kamphuis, J. H. (2015). Development and preliminary psychometric evaluation of a brief selfreport questionnaire for the assessment of the DSM-5 level of personality functioning scale: The LPFS brief form (LPFS-BF). Personality Disorder, 7(2), 192-197.

32. Oltmanns, T.F., Turkheimer, E. (2006). Perceptions of self and others regarding pathological personality traits. In: R.F. Krueger, J, Tackett (Eds.), Personality and psychopathology: Building bridges, (pp. 71-111). New York: Guilford
33. Bjureberg, J., Ljótsson, B., Tull, M. T., Hedman, E., Sahlin, H., Lundh, L. G., et al. (2016). Development and validation of a brief version of the difficulties in emotion regulation scale: The DERS16. Journal of Psychopathological and Behavioral Assessment, 38(2), 284-296.

34. Keetharuth, A. D., Brazier, J., Connell, J., Bjorner, J. B., Carlton, J., Taylor Buck, E., et al. (2018). Recovering quality of life (ReQoL): A new generic self-reported outcome measure for use with people experiencing mental health difficulties. British Journal of Psychiatry, 212(1), 42-49.

35. Posner, K., Brown, G. K., Stanley, B., Brent, D. A., Yershova, K. V., Oquendo, M. A., et al. (2011). The columbia-suicide severity rating scale: Initial validity and internal consistency findings from three multisite studies with adolescents and adults. American Journal of Psychiatry, 168(12), 1266-1277.

36. Ratey, J. J., \& Gutheil, C. M. (1991). The measurement of aggressive behavior: Reflections on the use of the overt aggression scale and the modified overt aggression scale. Journal of Neuropsychiatry and Clinical Neurosciences, 3(2), S57-S60.

37. Papadopoulou, M., Stasi, S., Bakalidou, D. et al. (2020). Psychometric properties of the 12-item world health organization disability assessment schedule (WHODAS 2.0) in adult patients with motor disabilities. Journal of Developmental and Physical Disabilities, 32, 801-819

38. Ravens-Sieberer, U., Erhart, M., Rajmil, L., Herdman, M., Auquier, P., Bruil, J., et al. (2010). Reliability, construct and criterion validity of the KIDSCREEN-10 score: A short measure for children and adolescents' well-being and health-related quality of life. Quality of Life Research, 19(10), 1487-1500.

39. Johnston, K., Lawrence, S., Dodds, N., Yu, L., Daley, D., \& Pilkonis, P. (2016). Evaluating PROMIS ${ }^{\circledR}$ instruments and methods for patient-centered outcomes research: Patient and provider voices in a substance use treatment setting. Quality of Life Research, 25(3), 615-624.

Publisher's Note Springer Nature remains neutral with regard to jurisdictional claims in published maps and institutional affiliations. 